\section{How brains develop} considers writing a book. But what type of book? A textbook may be most familiar, but increasingly, researchers are trying their hand at and gaining credit for penning books aimed at the popular market. An additional challenge is choosing a topic of broad relevance and then balancing the scientific content with the need to keep the reader engaged.

Two new books reflect this conflict of communication. Joan Stiles's The Fundamentals of Brain Development and Charles Fernyhough's The Baby in the Mirror both tackle the biological basis of child development, but with a vastly different style, content and target audience. The former is a standard science textbook, whereas the latter is an intimate diary of fatherhood.

Stiles is clear in her objective to provide "an overview of the fundamentals of mammalian brain development" that is "directed at students and investigators working in the behavioural sciences who wish to understand the biological underpinnings of the complex changes observed in perceptual, cognitive, affective and social development".

Fernyhough prefers a more personal approach. The book jacket tells us that it is "an authoritative, intimate and beautifully written account of a young child's development" that "will allow parents and all those fascinated by child development to understand the extraordinary journey a child makes during the momentous first three years of life".

The Fundamentals of Brain Development, as Stiles intended, is a timely survey of developmental neurobiology. Neuronal differentiation, migration, plasticity, sensitive periods, intrinsic factors and a discussion of experiential environments are all here. It is detailed, beautifully illustrated, thoroughly researched and should become a standard for any researcher who needs to look to neurobiological explanations to account for early developmental changes.

Her book also tackles the big issues. What does it mean to talk about innate knowledge? How could genes code for such information? Importantly, should behavioural scientists be concerned about biology at all? Stiles's approach is detached and deliberately impersonal.

By contrast, Fernyhough wants to talk to his audience. The Baby in the Mirror documents
At some point in their career, every scientist brain development in early childhood through his eyes, as a father who also happens to be a psychologist. His book is a diary of raising his daughter, punctuated with meticulous notes. Fernyhough points out the milestones of development that could be so easily missed when embedded in everyday acts of play. Where some see a hapless infant trying to grasp a toy, Fernyhough identifies an emerging perception-action system supported by maturing cortical mechanisms that enable object manipulation and representation. He weaves together psychology, neuroscience and philosophy to explain

Like Stiles, Fernyhough tackles the big questions, but at a level that a lay reader can follow. It works because Fernyhough is a passionate individual who is comfortable sharing his private life. He admits to bouts of melancholy, self-doubt and self-analysis, and takes pains to convey the meaning and emotional the developmental stages. timbre that his daughter brings to his life. Yet this openness can sometimes make the reader feel intrusive.

The language is often vivid, for instance: "Grief was a planet I would slingshot around in the early hours, a galactic outpost so toxic that it was only safe to go past it in a state of suspended animation." Although Fernyhough's poetics may be a device to keep the reader emotionally engaged, it creates uneven balance. The reader is often left reeling by the jump from narrative, to angst, to neuroscience. Sometimes, undefined jargon creeps in and is used without sufficient explanation. His discussion of sleep, dreaming and cot death is intriguing, but will be alarming to new parents who agonize over this subject.

By comparison with the rigour of The Fundamentals of Brain Development, it is easy to find flaws in The Baby in the Mirror. But it has ambition in bringing this science to the public. Although seemingly very different, both books achieve their goals, bringing neuroscience to their respective audiences.

Bruce M. Hood is professor of developmental psychology at the Bristol Cognitive Development Centre, Department of Experimental Psychology, University of Bristol, Bristol BS8 1TN, UK, and author of the forthcoming book The Supernatural Sense.

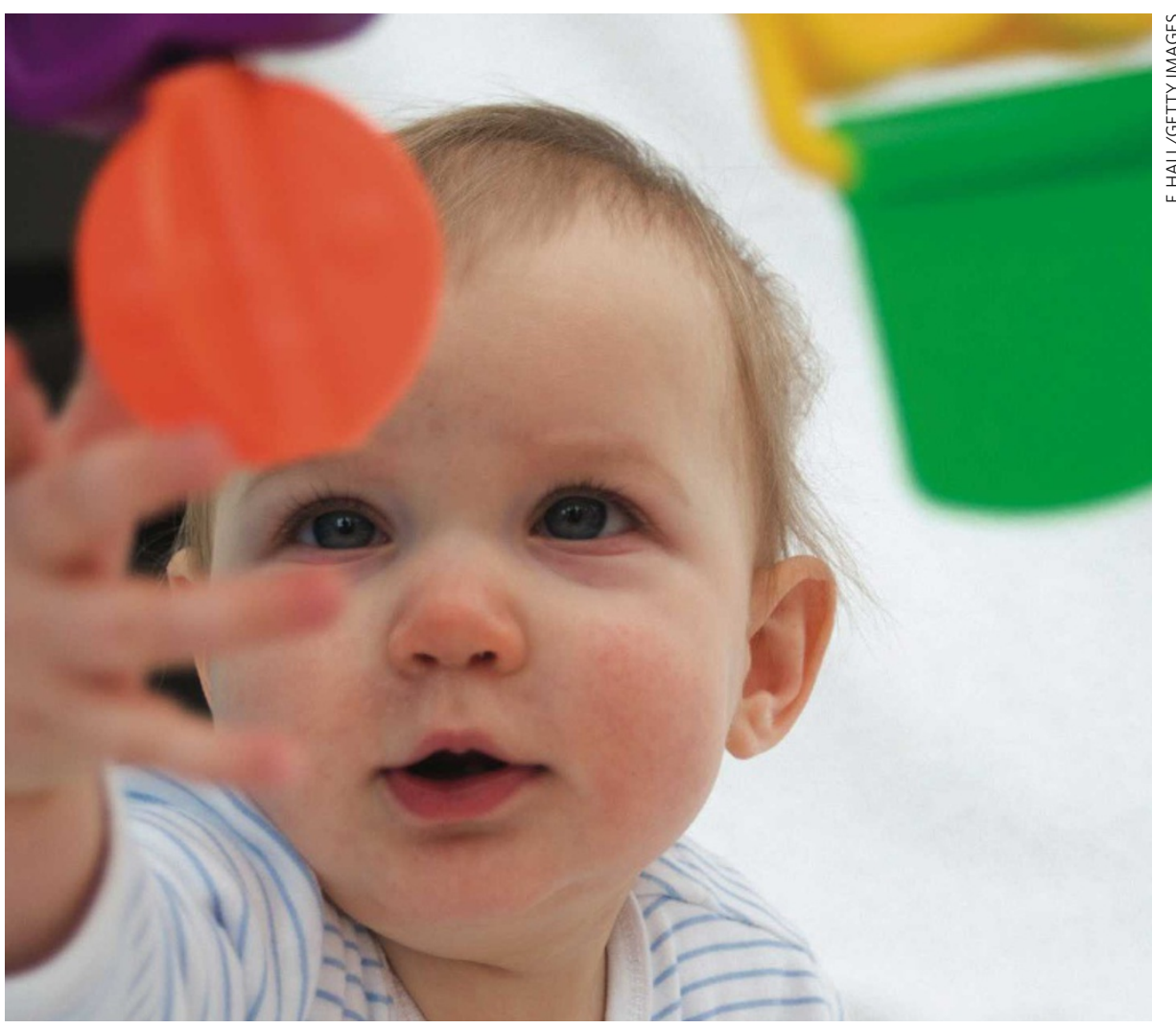

Simple acts of play, such as grasping a toy, enable development of a child's perception-action system. 